\title{
Fast and Non-destructive Pore Structure Analysis using Terahertz Time-Domain Spectroscopy
}

\author{
Daniel Markl ${ }^{\mathrm{a}, *}$, Prince Bawuah ${ }^{\mathrm{b}}$, Cathy Ridgway ${ }^{\mathrm{c}}$, Sander van den Ban ${ }^{\mathrm{d}}$, Daniel J \\ Goodwin $^{\mathrm{e}}$, Jarkko Ketolainen ${ }^{\mathrm{b}}$, Patrick Gane ${ }^{\mathrm{c}, \mathrm{f}}$, Kai-Erik Peiponen ${ }^{\mathrm{g}}$, J Axel Zeitler ${ }^{\mathrm{a}}$ \\ ${ }^{a}$ Department of Chemical Engineering and Biotechnology, University of Cambridge, Philippa Fawcett \\ Drive, CB3 OAS, Cambridge, UK \\ ${ }^{6}$ School of Pharmacy, Promis Centre, University of Eastern Finland, P.O. Box 1617, 70211, Kuopio, \\ Finland \\ ${ }^{c}$ Omya International AG, 4665, Oftringen, Switzerland \\ ${ }^{d}$ GSK Research and Development, New Frontiers Science Park, 3rd Avenue, CM19 5AW, Harlow, UK \\ ${ }^{e}$ GSK Global Manufacturing and Supply, Priory Street, SG12 ODJ, Ware, UK \\ ${ }^{f}$ School of Chemical Engineering, Department of Bioproducts and Biosystems, Aalto University, 00076 \\ Aalto, Helsinki, Finland \\ ${ }^{g}$ Institute of Photonics, University of Eastern Finland, P.O. Box 111, 80101 Joensuu, Finland
}

\begin{abstract}
Pharmaceutical tablets are typically manufactured by the uni-axial compaction of powder that is confined radially by a rigid die. The directional nature of the compaction process yields not only anisotropic mechanical properties (e.g. tensile strength) but also directional properties of the pore structure in the porous compact. This study derives a new quantitative parameter, $S_{a}$, to describe the anisotropy in pore structure of pharmaceutical tablets based on terahertz time-domain spectroscopy measurements. The $S_{a}$ parameter analysis was applied to three different data sets including tablets with only one excipient (functionalised calcium carbonate), samples with one excipient (microcrystalline cellulose) and one drug (indomethacin), and a complex formulation (granulated product comprising several excipients and one drug). The overall porosity, tablet thickness, initial particle size distribution as well as the granule density were all found to affect the significant structural anisotropies that were observed in all investigated tablets. The $S_{a}$ parameter provides new insights into the microstructure of a tablet and its potential was particularly demonstrated for the analysis of formulations comprising several components. The results clearly indicate that material attributes, such as particle size and granule density, cause a change of the pore structure, which, therefore, directly impacts the liquid imbibition that is part of the disintegration process. We show, for the first time, how the granule density impacts the pore structure, which will also affect the
\end{abstract}


performance of the tablet. It is thus of great importance to gain a better understanding

35 of the relationship of the physical properties of material attributes (e.g. intragranular

36 porosity, particle shape), the compaction process and the microstructure of the finished

37 product.

38 Keywords: Pharmaceutical tablet, terahertz, pore structure, microstructure,

39 anisotropy, disintegration

\section{1. Introduction}

${ }_{41}$ Powder compaction is a central process in pharmaceutical tablet manufacturing. Dur-

42 ing compaction, force is applied on an aggregate of mixed particles of excipient and active

43 pharmaceutical ingredient (API) to transform the powder bed into a porous compact of

44 a well-defined shape. The compaction process involves the rearrangement, plastic and

45 elastic deformation, as well as fragmentation of particles. As a result, individual par-

46 ticles come into sufficiently close proximity with one other to establish interparticulate

47 attractive forces or bonds (Nyström et al., 2008). The magnitude of the particle-particle

48 bonding forces depends on the underlying physical mechanism of bonding between the

49 individual particles as well as on the surface areas of interparticulate contacts. The

50 resulting interparticulate bonds do not only govern the tensile strength of the powder

51 compact, but they play a key role in the tablet disintegration process. A tablet only

52 disintegrates when sufficiently many interparticulate bonds are broken. This process

${ }_{53}$ is typically achieved or facilitated by the stress that is generated from the swelling of

${ }_{54}$ specifically designated excipient particles (Markl and Zeitler, 2017). A wide range of

55 pharmaceutical excipient particles begin to swell when they come in contact with water

56 or physiological fluids in the body. The swelling and subsequent disintegration of the

57 powder compact is thus driven by the rate at which liquid penetrates into the porous

58 tablet. The liquid uptake rate is directly affected by the pore space in the tablet, which

59 is formed during powder compaction (Yassin et al., 2015a,b). It is well-established in

60 other fields that the liquid imbibition process is quite complex and cannot be described

${ }_{61}$ accurately only by the overall total porosity (Berg, 2014) but that it is considerably

*Corresponding author's electronic address: dm733@cam.ac.uk 
impacted by other characteristic properties of the pore structure, such as their pore connectivity, constrictivity and tortuosity as well as pore wall roughness Schoelkopf (2000); Liu et al. (2014). It is these properties that are particularly affected by anisotropy in pore structure as they are defined by the compaction process.

Powder compaction is inherently anisotropic in nature as the powder particles are confined radially by a rigid die whilst they are compressed axially by the moving punch (Moe and Rippie, 1997). Unsurprisingly, this yields directionally dependent mechanical properties of the finished tablet. It is well-known that the tensile strength varies significantly between the axial and radial direction (Mullarney and Hancock, 2006) as well as that the punch geometry affects the density distribution within a tablet Eiliazadeh et al. (2003, 2004). Such anisotropic mechanical properties explain common failure modes that are encountered during tablet manufacturing such as capping and delamination. Besides the impact on the mechanical properties, uni-axial compression also affects the pore structure within a tablet. Thus far there is a much better understanding of the directional mechanical properties in tablets and much less is known with regard to the anisotropy of the void space. This is not too surprising given that it is much harder to measure and quantify. The most common methods for pore characterisation, namely mercury porosimetry and helium pycnometry, cannot resolve the directionality of the pores.

One method that can provide such information is X-ray computed microtomography $(\mathrm{X} \mu \mathrm{CT})$. It was recently demonstrated that $\mathrm{X} \mu \mathrm{CT}$ can be used to analyse the orientation of pores by representing individual pores by their eigenvectors (Markl et al., 2017b). The first eigenvector is defined in the direction of the main axis of a pore and its angle relative to the global coordinate system of the macroscopic tablet provides a measure of the orientation of the pore. Another very promising method to study the pore structure of pharmaceutical tablets is terahertz time-domain spectroscopy (THz-TDS). THz-TDS can be employed to measure the effective refractive index of a sample in a non-destructive manner (Bawuah et al., 2016b; Markl et al., 2017a,b). The effective refractive index is a function of the fill fractions of each constituent as well as of the total porosity.

It can therefore be used to determine the porosity of a tablet within seconds. The measurement speed is clearly an advantage of THz-TDS (seconds) compared to $\mathrm{X} \mu \mathrm{CT}$ 
(hours). Although the terahertz method cannot provide information about individual pores, by using polarised terahertz radiation, it is possible to analyse the directional properties of the pore structure as introduced recently (Bawuah et al., 2016a).

This study presents a rigorous development of a new structural parameter, $S_{a}$, to characterise anisotropic pore structures using THz-TDS based on the method originally developed by Bawuah et al. (2016a). The $S_{a}$ parameter is derived for a two-phase system (air filled pores and one solid material) and then extended to multi-phase porous media (air filled pores and several different components). This parameter is applied to characterise a simple formulation, a three-phase system and eventually a complex commercial formulation.

\section{Theory}

Using THz-TDS it is possible to measure the effective refractive index, $n_{\mathrm{eff}}$, of a porous medium, which is related to the effective permittivity by $\varepsilon_{\text {eff }}=n_{\text {eff }}^{2}$. Effective medium theory or zero-porosity approximation (ZPA) can be applied to calculate the porosity from $\varepsilon_{\text {eff }}$ or $n_{\text {eff }}$ (Markl et al., 2017b). Besides determining the total porosity, it is possible to gain insight into the directionality of the pores by exploiting the polarisation of the terahertz wave. The terahertz plane wave's electric field vector, $\mathbf{E}$, is in the direction perpendicular to the propagation direction, $\mathbf{k}$, of the terahertz pulse. The specific direction of the terahertz electric field can thus be used to analyse anisotropic characteristics of the pores or the material of the probed medium. We begin with deriving the lower and upper limits of $\varepsilon_{\text {eff }}$ on the basis of a theoretical model of the porous medium that was first introduced by Wiener (1912). This model is then combined with the experimental terahertz data to define a new characteristic parameter, $S_{a}$, that is a measure of the degree of anisotropy of a pore structure. Initially, this concept is derived for a two-phase system (one solid material and air voids) and then generalised for multiphase systems. Throughout this study we assume that scattering and dispersion effects are negligible as well as that the fill fraction(s), i.e. porosity and fractions of each constituent, are known. 


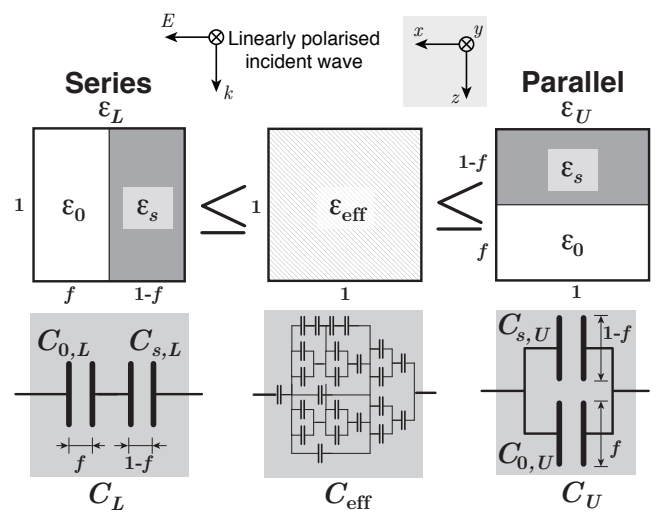

Figure 1: Schematic of the equivalent electric circuits for the propagation of a terahertz pulse in the $z$-direction through a two-phase porous compact composed of air and solid phase. The extreme cases are represented by the arrangement in series (left, lower Wiener bound) and in parallel (right, upper Wiener bound). The permittivity of air is $\varepsilon_{0}=1$ and that of the solid material is $\varepsilon_{s}$. The effective permittivity, $\varepsilon_{\text {eff }}$, for an actual sample always falls between the lower, $\varepsilon_{L}$, and the upper, $\varepsilon_{U}$, limits defined by the Wiener bounds. Its equivalent circuit is a combination of series and parallel capacitors. The dimensions of the capacitors, which are not provided in the schematic, are all set to unity.

\subsection{Extreme Cases of the Pore Structure: Wiener Bounds}

The permittivity of a porous medium can be calculated by considering a unit cell in which a repeating layered structure is used to reflect the pore structure. In this representation the pore space is described as air layers that are separated by solid matrix layers. The dimensions of the layers are much smaller than the wavelength of the terahertz waves, which are propagating parallel to the plane of the unit cell. Two extreme cases define the minimum and maximum of $\varepsilon_{\text {eff }}$ : a serial and a parallel arrangement of the solid material, $\varepsilon_{s}$, and the air, $\varepsilon_{0}$, respectively (Figure 1). The direction of the terahertz electric field defines the type of arrangement (e.g. the material and air are in series when they are arranged along $\mathbf{E}$ ). The description of $\varepsilon_{\text {eff }}$ can be performed analogous to electrical circuitry using capacitors to represent the material and air (Kadlec et al., 2008).

The relative capacitance of the serial $\left(C_{L}\right)$ and parallel $\left(C_{U}\right)$ configuration can thus 
be calculated by

$$
\begin{aligned}
\frac{1}{C_{L}} & =\frac{1}{C_{0, L}}+\frac{1}{C_{S, L}} \\
C_{L} & =\frac{C_{0, L} C_{S, L}}{C_{0, L}+C_{S, L}} \\
C_{U} & =C_{0, U}+C_{S, U} .
\end{aligned}
$$

In general a capacitance is defined as $C=\varepsilon \frac{A}{w}$ with $\varepsilon, A, w$ as the permittivity of the dielectric medium between the two plates, the area of the conductive plates and the distance between the two plates, respectively. Figure 1 depicts only the dimensions that deviate from unity and therefore either the area of the conductive plates or the distance between the two plates is assumed to be unity. A unit length represents the amplitude of the $\mathbf{E}$ vector. Furthermore, the influence of the $x-y$ geometry of the tablet on the terahertz measurement is negligible as the cross-section of the beam is much smaller than the dimensions of the tablet (i.e. the diameter of the tablet). In other words, the electric field decays in a distance much less than the radius of the tablet so that the dependence on the lateral geometry is negligible. The capacitance in the Eqs. 1 and 2 as well as in Figure 1 can thus be defined as $C_{0, L}=\varepsilon_{0} \frac{1}{f}, C_{s, L}=\varepsilon_{s} \frac{1}{(1-f)}, C_{0, U}=\varepsilon_{0} f$ and $C_{s, L}=\varepsilon_{s}(1-f)$. The intrinsic permittivity $\varepsilon_{s}$ is a material property and it refers to the skeletal material of the particle, i.e. the permittivity of the material in the absence of any intraparticle pores. $\varepsilon_{0}=1$ is taken to approximate the permittivity of ambient air $(\varepsilon=1.0006)$. The extreme cases, Eqs. 1 and 2, can now be expressed in terms of their permittivities as

$$
\begin{aligned}
\varepsilon_{L} & =\frac{\varepsilon_{s}}{f\left(\varepsilon_{s}-1\right)+1} \\
\varepsilon_{U} & =f+(1-f) \varepsilon_{s}=f\left(1-\varepsilon_{s}\right)+\varepsilon_{s}
\end{aligned}
$$

with $\varepsilon_{L}=C_{L}$ and $\varepsilon_{U}=C_{U}$. Eqs. 3 and 4 are well known as the lower and upper limits of the Wiener bounds for a two-phase porous medium (Tuononen et al., 2010; Bawuah et al., 2016a). The true value of the effective permittivity must always fall within the Wiener bounds $\left(\varepsilon_{L} \leq \varepsilon_{\text {eff }} \leq \varepsilon_{U}\right)$ for arbitrary shapes of the pores and the solid material.

As outlined above, the Wiener bounds represent the extreme cases of either a fully parallel or in series arrangement of the solid material and the pores. However, in reality 


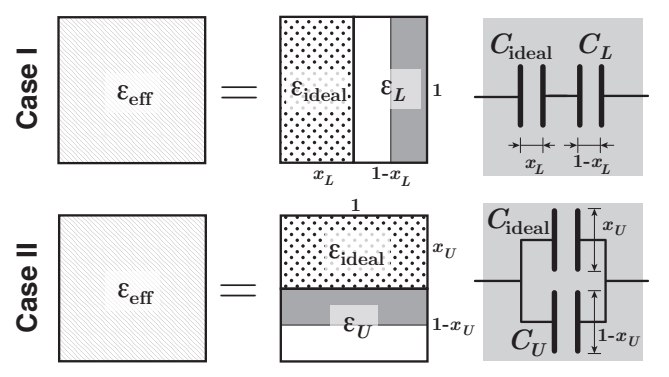

Figure 2: Representation of the two cases as electric circuits. The ideal permittivity, $\varepsilon_{\text {ideal }}$, is given by the underlying model (e.g. effective medium theory, ZPA) used to relate $n_{\text {eff }}$ from the terahertz measurements to the porosity. $x_{L}$ and $x_{U}$ are fractional dimensions to express $C_{\text {idela }}, C_{L}$ and $C_{U}$ as a function of their respective permittivities.

the pore architecture in a pharmaceutical tablet forms a complex structure that can be approximated by a combination of parallel and serial circuits (Figure 1). Bawuah et al. (2016a) used the concept of Wiener bounds and adapted a model from effective heat conductivity (Krischer and Kast, 1978) to study the structure of pharmaceutical tablets. The effective permittivity was used in conjunction with both the upper and the lower bound permittivities of the Wiener limits model to derive a structure parameter $(S)$ for porous pharmaceutical tablets:

$$
\begin{aligned}
\frac{1}{\varepsilon_{\mathrm{eff}}} & =\frac{S}{\varepsilon_{L}}+\frac{1-S}{\varepsilon_{U}} \\
S & =\frac{1}{\varepsilon_{U}-\varepsilon_{L}}\left(\frac{\varepsilon_{U} \varepsilon_{L}}{\varepsilon_{\mathrm{eff}}}-\varepsilon_{L}\right) .
\end{aligned}
$$

\subsection{Alternative Definition of Structural Parameter}

The $S$ parameter as defined in Eq. 5 strongly depends on the porosity and it is thus limited to study structural changes of samples of the same porosity. We therefore propose an alternative definition, the $S_{a}$ parameter, which enables the comparison of structural changes for samples with different porosities.

This reused structural parameter $S_{a}$ is defined to be 0.5 for the case of a completely random arrangement of solid material and pores. This means that the measured $\varepsilon_{\text {eff }}$ is equal to the theoretical/ideal permittivity, $\varepsilon_{\text {ideal }}$. $\varepsilon_{\text {ideal }}$ can be calculated from the known porosity, $f$, and the intrinsic permittivity, $\varepsilon_{s}$, of the material using either an effective 
medium approximate (EMA) or ZPA. The proposed $S_{a}$ model considers two cases: i)

$\varepsilon_{\text {eff }}<\varepsilon_{\text {ideal }}$, and ii) $\varepsilon_{\text {eff }} \geq \varepsilon_{\text {ideal }}$ (Figure 2).

The effective permittivity is represented by the ideal permittivity in conjunction with either a serial (Case I) or a parallel (Case II) arrangement as the boundary of each extreme case (Figure 1). In Case I this leads to

$$
\begin{aligned}
\frac{1}{\varepsilon_{\text {eff }}} & =\frac{x_{L}}{\varepsilon_{L}}+\frac{1-x_{L}}{\varepsilon_{\text {ideal }}} \\
x_{L} & =\frac{\varepsilon_{L}\left(\varepsilon_{\text {ideal }}-\varepsilon_{\text {eff }}\right)}{\varepsilon_{\text {eff }}\left(\varepsilon_{\text {ideal }}-\varepsilon_{L}\right)} .
\end{aligned}
$$

Case II can be expressed as

$$
\begin{aligned}
\varepsilon_{\text {eff }} & =x_{U} \varepsilon_{U}+\left(1-x_{U}\right) \varepsilon_{\text {ideal }} \\
x_{U} & =\frac{\varepsilon_{\text {eff }}-\varepsilon_{\text {ideal }}}{\varepsilon_{U}-\varepsilon_{\text {ideal }}} .
\end{aligned}
$$

$x_{L}$ and $x_{U}$ are the fractional dimension of the representative capacitors. These fractional dimensions range from 0 to 1 , where 0 yields $\varepsilon_{\text {eff }}=\varepsilon_{\text {ideal }}$ and 1 corresponds to the extreme case. The $S_{a}$ parameter combines these two cases by

$$
S_{a}= \begin{cases}0.5\left(1-x_{L}\right) & \text { for } \varepsilon_{\mathrm{eff}}<\varepsilon_{\text {ideal }} \\ 0.5\left(1+x_{U}\right) & \text { for } \varepsilon_{\mathrm{eff}} \geq \varepsilon_{\text {ideal }}\end{cases}
$$

Consequently, $S_{a}$ ranges from 0 to 1 and it indicates the degree of parallel and serial arrangements of pore/solid material structures in a sample.

Rather than in transmission, the $S_{a}$ as well as the $S$ parameter can also be applied to study the tablet structure on the basis of terahertz reflection measurements. A reflection setting could be beneficial for imaging applications or in-process control of the tablet quality where a transmission setup is not feasible. The change of the measurement configuration only affects the calculation of $n_{\text {eff }}$ from the reflected terahertz waveform (Jepsen et al., 2007), but it does not require a modification of the definitions of the structural parameters or the Wiener bounds.

\subsection{Multi-Phase Systems}

The $S_{a}$ and $S$ parameters, as defined above, can be applied to reflect the arrangement of the pores in more complex tablet matrices. For a formulation of $J$ constituents in the 
powder compact, $J$ solid materials are considered in the calculation of the Wiener bounds using the general definition:

$$
\begin{gathered}
\varepsilon_{L}=\frac{1}{f+\sum_{j=1}^{J} \frac{x_{j}}{\varepsilon_{j}}} \\
\varepsilon_{U}=f+\sum_{j=1}^{J} x_{j} \varepsilon_{j}
\end{gathered}
$$

with $x_{j}$ as the fill fraction of component $j$. Using Eqs. 9 and 10 to determine the $S_{a}$ parameter allows to study the arrangement and structure of all components including pores.

This, however, does not enable the dissociation of the pore/solid material arrangement from that of the different components to each other and to the pores.

In order to study only the arrangement of the pores with respect to the solid phase, we propose to consider all solid constituents as one solid material, which is described by the lumped intrinsic permittivity, $\varepsilon_{s, \text { lumped }}$. The lower and upper bounds are thus defined as

$$
\begin{aligned}
\varepsilon_{L} & =\frac{1}{f+\sum_{j=1}^{J} \frac{x_{j}}{\varepsilon_{j}}} \\
& =\frac{1}{f+\frac{x_{\text {rest }}}{\varepsilon_{s, \text { lumped }}}}=\frac{1}{f+\frac{1-f}{\varepsilon_{s, \text { lumped }}}} \\
\varepsilon_{U} & =f+\sum_{j=1}^{J} x_{j} \varepsilon_{j} \\
& =f+x_{\text {rest }} \varepsilon_{s, \text { lumped }}=f+(1-f) \varepsilon_{s, \text { lumped }}
\end{aligned}
$$

with $x_{\text {rest }}=1-f$. The multi-phase system can now be treated like a two-phase system where the pores are embedded in an effective medium. The theories developed for two-phase systems, such as the $S_{a}$ parameter (Eq. 8), can be applied to porous media consisting of several components using Eqs. 11 and 12.

\subsection{Extreme Values of the Wiener Bounds}

As discussed above, the Wiener bounds are used to gain insights into the anisotropic structure of pharmaceutical tablets. The $S_{a}$ and $S$ parameter depend on how close the upper and lower bounds of the Wiener limits are to each other and how they are related 
to the porosity. The maximum separation of the Wiener bounds for two-phase compacts is studied on the basis of the function

$$
F(f)=\varepsilon_{U}(f)-\varepsilon_{L}(f) .
$$

Combining Eqs. 3, 4 and 13 gives

$$
F(f)=f\left(1-\varepsilon_{s}\right)+\varepsilon_{s}-\frac{\varepsilon_{s}}{f\left(\varepsilon_{s}-1\right)+1} .
$$

The maximum separation condition for the Wiener bounds can be determined by forming the derivative with respect to the porosity:

$$
F^{\prime}(f)=\left(1-\varepsilon_{s}\right)+\frac{\left(\varepsilon_{s}-1\right) \varepsilon_{s}}{\left[f\left(\varepsilon_{s}-1\right)+1\right]^{2}} .
$$

The porosity maximising the separation of the Wiener bounds, $f_{\max }$, can be calculated by setting $F^{\prime}\left(f_{\max }\right)=0$, which yields

$$
\begin{aligned}
\frac{\left(\varepsilon_{s}-1\right) \varepsilon_{s}}{\left[f_{\max }\left(\varepsilon_{s}-1\right)+1\right]^{2}} & =\left(\varepsilon_{s}-1\right) \\
f_{\max } & =\frac{\sqrt{\varepsilon_{s}}-1}{\varepsilon_{s}-1} .
\end{aligned}
$$

We can now replace the permittivity by the refractive index $\left(n_{s}=\sqrt{\varepsilon_{s}}\right)$ in Eq. 16 and $f_{\max }$ can then be expressed as

$$
f_{\max }=\frac{n_{s}-1}{n_{s}^{2}-1}=\frac{1}{n_{s}+1} .
$$

Eq. 17 gives the porosity which maximises the separations of the Wiener bounds for a two-phase system and also for a multi-phase system as described by Eqs. 11 and 12. This can be confirmed by forming the second derivative $F^{\prime \prime}(f)$ :

$$
\begin{aligned}
F^{\prime \prime}(f) & =-\frac{2\left[f\left(\left(\varepsilon_{s}-1\right)+1\right]\left(\varepsilon_{s}-1\right)^{2} \varepsilon_{s}\right.}{\left[f\left(\varepsilon_{s}-1\right)+1\right]^{4}} \\
F^{\prime \prime}\left(f_{\max }\right) & =-\frac{2\left(\varepsilon_{s}-1\right)^{2} \varepsilon_{s}}{\left[f_{\max }\left(\varepsilon_{s}-1\right)+1\right]^{3}}<0
\end{aligned}
$$

${ }_{167}$ This inequality is always true as $\varepsilon_{s}>1$, which confirms that Eq. 17 yields a maximum. 


\section{Materials and Methods}

\subsection{Materials}

In the following we present data from several different formulations ranging from a simple one with only one excipient to a complex formulation consisting of several excipients and an API. The terahertz transmission data of these formulations were already reported previously as indicated below, but we use these datasets to demonstrate how the concept of $S_{a}$ can be applied to better understand the microstructure of pharmaceutical tablets in general and the anisotropic pore structure in particular.

The porosity was calculated by relating the bulk density, $\varrho_{b}$, to the known true density, $\varrho_{t}$, of the used materials:

$$
f=1-\frac{\varrho_{b}}{\varrho_{t}}
$$

The bulk density of flat-faced tablets (first two sets of samples, M01 and M02) was determined by $\varrho_{b}=W /\left(\pi\left(\frac{D}{2}\right)^{2} H\right)$ with $W, D$ and $H$ as the weight, diameter and thickness of the tablet. The third set of samples (M03) were tablets compacted by dual radius punches and the bulk density was thus calculated by $\varrho_{b}=W /\left(0.25 H_{\text {fill } \pi D^{2}+V_{\text {cup }}}\right)$, where $H_{\text {fill }}$ and $V_{\text {cup }}$ are the fill depth and the tablet cup volume, respectively.

The first set of samples (henceforth referred to as M01) consisted of pure functionalised calcium carbonate (FCC, Omyapharm ${ }^{\circledR}$, Omya International AG, Oftringen,

Table 1: Summary of the M01 samples, namely pure FCC tablets with target porosities from $45 \%$ to 67\%. More details about these tablets were reported previously in Markl et al. (2017b). Each batch consisted of 15 tablets and the properties listed are averaged values for each batch. The porosity was calculated using Eq. 19 with a true density of $2.96 \mathrm{~g} \mathrm{~cm}^{-3}$.

\begin{tabular}{cccc} 
Batch ID & Thickness & Weight & Porosity \\
& $H$ & $W$ & $f$ \\
& $(\mathrm{~mm})$ & $(\mathrm{mg})$ & $(-)$ \\
\hline B01 & 1.67 & 217 & 0.45 \\
B02 & 1.64 & 190 & 0.50 \\
B03 & 1.63 & 168 & 0.55 \\
B04 & 1.62 & 147 & 0.61 \\
B05 & 1.61 & 122 & 0.67 \\
\hline
\end{tabular}


Switzerland) particles. Details about this set of samples are provided in Markl et al. (2017b) and a summary is given in Table 1. The FCC powder was compacted to flatfaced tablets with a target diameter of $10 \mathrm{~mm}$ using a compaction simulator (PuuMan Ltd, Kuopio, Finland). The highly-porous nature of FCC allowed the compaction of tablets with porosities ranging from $45 \%-67 \%$.

The second set of samples consisted of microcrystalline cellulose (MCC, Avicel PH101, FMC BioPolymer, Philadelphia, USA) and indomethacin (Hangzhou Dayangchem Co. Ltd, Hangzhou, China), as reported in Bawuah et al. (2016b) and Ridgway et al. (2017). These samples were varied in terms of $\mathrm{API} / \mathrm{MCC}$ concentrations, tablet thickness and porosity (Table 2). Several different subsets were produced by a compaction simulator (PuuMan Ltd, Kuopio, Finland), where each flat-faced tablet had a diameter of $13 \mathrm{~mm}$.

Another set of samples consists of 18 batches from a production-scale design of experiments (DoE) (Table 3). The tablet formulation was kept constant throughout all batches. Each tablet consisted of a micronised poorly water soluble drug substance which was combined with lactose monohydrate, MCC, hypromellose and croscarmellose sodium by a high shear wet granulator. A fluid-bed dryer was employed to dry the granules prior to blending them with extra-granular croscarmellose sodium and magnesium stearate. This blend was then compacted by a Fette 2090 tablet press (Fette Compacting GmbH, Schwarzenberg, Germany, fitted with 43 stations) to curved-face tablets using dual radius punches with a diameter of $10.5 \mathrm{~mm}$. A split-plot DoE was used to study the effect of three process variables (water amount, wet massing time and water addition rate) of the granulation and one compaction factor (tablet breaking force) on the disintegration and dissolution performance of the tablets. More details about these samples are provided in Markl et al. (2017a) and van den Ban and Goodwin (2017).

\subsection{Terahertz Time-Domain Spectroscopy}

The effective refractive index of each sample was determined from terahertz transmission measurements. A Terapulse 4000 spectrometer (TeraView Ltd, Cambridge, UK) was used for the samples M01 and M03. The measurements were performed in a transmission chamber, which was purged with dry nitrogen gas. 60 waveforms were co-averaged and the total measurement time was about $1.5 \mathrm{~min}$ for M01 and M03. $n_{\text {eff }}$ was determined for every tablet by analysing the frequency-dependent refractive index and selecting the 
214 refractive index at $1 \mathrm{THz}$. More details about the measurement procedure are provided

Table 2: Properties of the M02 samples. The concentration of indomethacin, $\phi$, was varied from $0 \%$ to $15 \%$ and the fraction of MCC was adjusted accordingly. Each subset (labelled with S01-S08) consisted of 5 tablets. The porosity, $f$, was calculated using Eq. 19 with a true density of $1.56 \mathrm{~g} \mathrm{~cm}^{-3} \mathrm{and}_{1.37 \mathrm{~g} \mathrm{~cm}}^{-3}$ for MCC and indomethacin, respectively.

\begin{tabular}{ccccc}
\hline Batch ID & API & Thickness & Weight & Porosity \\
& $\phi$ & $H$ & $W$ & $f$ \\
& $($ wt\% $)$ & $(\mathrm{mm})$ & $(\mathrm{mg})$ & $(-)$ \\
\hline B01-S01 & 10.00 & 3.03 & 342 & 0.46 \\
B01-S02 & 10.00 & 3.03 & 357 & 0.43 \\
B01-S03 & 10.00 & 3.02 & 371 & 0.41 \\
B01-S04 & 10.00 & 3.01 & 385 & 0.38 \\
B01-S05 & 10.00 & 3.01 & 400 & 0.36 \\
\hline B02-S01 & 10.00 & 2.74 & 361 & 0.36 \\
B02-S02 & 10.00 & 3.33 & 439 & 0.36 \\
B02-S03 & 10.00 & 3.63 & 476 & 0.36 \\
B02-S04 & 10.00 & 3.93 & 515 & 0.36 \\
\hline B03-S01 & 0.00 & 3.03 & 411 & 0.36 \\
B03-S02 & 3.75 & 3.02 & 403 & 0.36 \\
B03-S03 & 7.50 & 3.03 & 403 & 0.36 \\
B03-S04 & 8.75 & 3.00 & 402 & 0.36 \\
B03-S05 & 10.00 & 3.02 & 401 & 0.36 \\
B03-S06 & 11.25 & 3.04 & 401 & 0.36 \\
B03-S07 & 12.50 & 3.03 & 400 & 0.36 \\
B03-S08 & 15.00 & 3.04 & 400 & 0.36 \\
\hline B04-S01 & 9.00 & 2.74 & 406 & 0.28 \\
B04-S02 & 9.50 & 2.96 & 405 & 0.34 \\
B04-S03 & 10.00 & 3.28 & 406 & 0.40 \\
B04-S04 & 10.50 & 3.65 & 403 & 0.47 \\
B04-S05 & 11.00 & 3.95 & 404 & 0.51 \\
\hline & & & & \\
\hline
\end{tabular}


Table 3: Summary of properties of M03 samples. This set consisted of 18 different batches from a production-scale DoE. The porosity was calculated from the ratio between the bulk density and the true density $\left(\varrho_{t}=1.51 \mathrm{~g} \mathrm{~cm}^{-3}\right)$ of the formulation following Eq. 19. The granule density was computed by $\varrho_{g}=W /\left(0.25 H_{\text {fill }} \pi D^{2}+V_{\text {cup }}\right)$, where $H_{\text {fill }}$ is the fill depth, $D=10.5 \mathrm{~mm}$ is the tablet diameter and $V_{\text {cup }}$ is the tablet cup volume. More details about these batches are provided in Markl et al. (2017a) and van den Ban and Goodwin (2017).

\begin{tabular}{ccccc}
\hline $\begin{array}{c}\text { Batch } \\
\text { ID }\end{array}$ & Thickness & Weight & Porosity & $\begin{array}{c}\text { Granule } \\
\text { density }\end{array}$ \\
& $H$ & $W$ & $f$ & $\begin{array}{c}\text { granule } \\
\left(\mathrm{g} \mathrm{cm}^{-3}\right)\end{array}$ \\
\hline B01 & 5.27 & 399.6 & 0.17 & 0.60 \\
B02 & 5.10 & 401.0 & 0.13 & 0.60 \\
B03 & 5.31 & 400.2 & 0.18 & 0.59 \\
B04 & 5.21 & 399.5 & 0.15 & 0.63 \\
B05 & 5.06 & 398.8 & 0.11 & 0.59 \\
B06 & 5.15 & 399.0 & 0.14 & 0.64 \\
B07 & 4.97 & 399.3 & 0.08 & 0.63 \\
B08 & 4.94 & 400.8 & 0.09 & 0.63 \\
B09 & 5.13 & 399.4 & 0.13 & 0.64 \\
B10 & 4.85 & 399.7 & 0.07 & 0.69 \\
B11 & 4.86 & 399.1 & 0.06 & 0.59 \\
B12 & 4.84 & 400.3 & 0.06 & 0.60 \\
B13 & 4.87 & 402.3 & 0.07 & 0.69 \\
B14 & 4.82 & 399.3 & 0.05 & 0.64 \\
B15 & 4.86 & 401.5 & 0.07 & 0.69 \\
B16 & 4.88 & 400.9 & 0.07 & 0.64 \\
B17 & 4.67 & 399.4 & 0.01 & 0.64 \\
B18 & 4.73 & 401.6 & 0.02 & 0.64 \\
\hline
\end{tabular}

215

in Markl et al. (2017b) and Markl et al. (2017a) for M01 and M03, respectively. The samples M02 were measured using a custom-built terahertz spectrometer as described in Parrott et al. (2009) and Li et al. (2010). $n_{\text {eff }}$ is retrieved from the terahertz pulse delay 

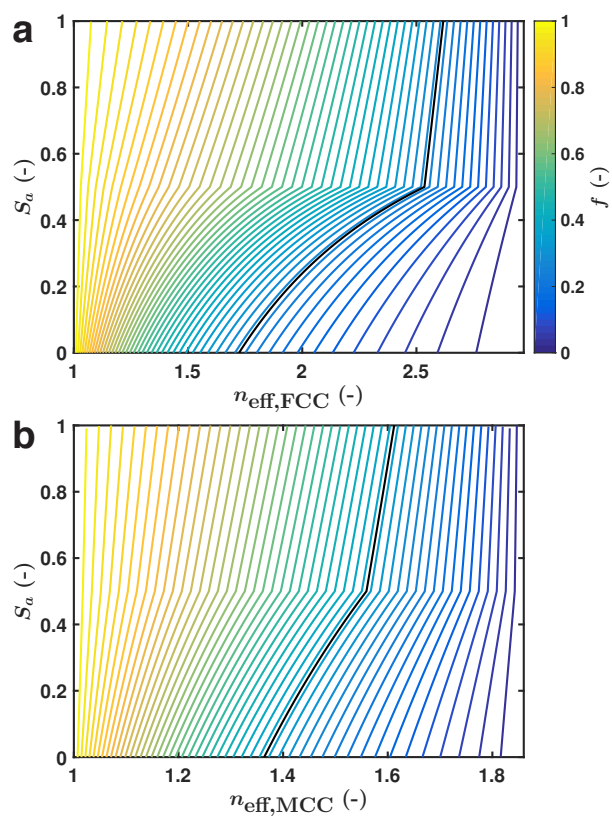

Figure 3: Simulation of the $S_{a}$ parameter for (a) FCC (M01) and (b) MCC (M02). The intrinsic refractive indices are given in Table 4 . The solid black line indicates the $S_{a}$ parameter at the porosity that maximises the Wiener bounds (Eq. 17).

difference between the sample and the reference pulse. Bawuah et al. (2016b) reported more details about the measurement procedure of these samples.

\section{Results}

\subsection{Simulations of the Extreme Cases and the $S_{a}$ Parameter}

The $S_{a}$ parameter was calculated for a range of $n_{\text {eff }}$ covering porosities of the porous medium from 0-1 and using the intrinsic refractive indices of FCC and MCC (Figure 3).

The discontinuity at $S_{a}=0.5$ is by definition the transition point between the two cases, i.e. serial and parallel arrangement of the constituents. The values of $S_{a}$ must always

be a real number between zero and one. Thus, for the two extremes of $S_{a}, S_{a}=1$ represents a porous medium with all its components purely arranged in parallel, whereas $S_{a}=0$ depicts a porous medium with components displaying ideal serial arrangement. 

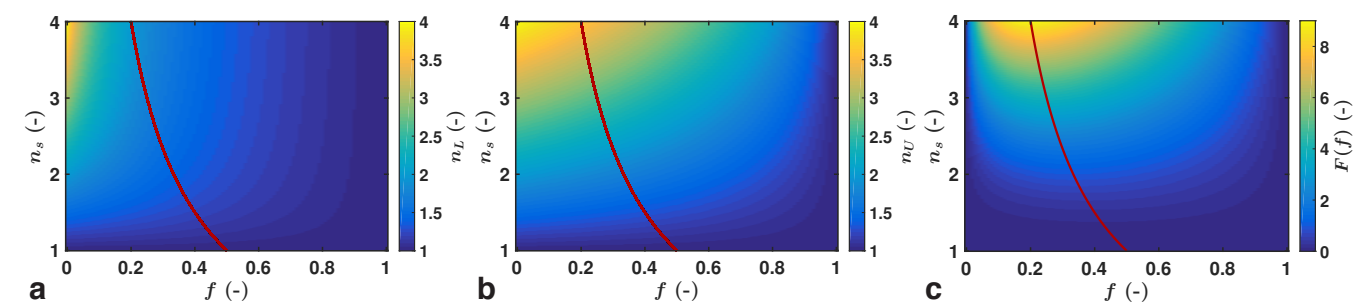

Figure 4: Intrinsic refractive index, $n_{s}$, as a function of porosity, $f$. (a) Lower Wiener bound $\left(n_{L}\right.$, Eq. 3). (b) Upper Wiener bound $\left(n_{U}\right.$, Eq. 4). (c) Separation function ( $F$, Eq. 13). The red solid line indicates the maximum separation of the Wiener bounds as given by Eq. 17 .

$S_{a}>0.5$ is linear due to the linear nature of Eqs. 4 and 7, whereas $S_{a}$ exhibits a nonlinear relationship with $n_{\text {eff }}$ for $S_{a}<0.5$ as evident in Eqs. 3 and 6 . The non-linear relationship between $S_{a}$ and $n_{\text {eff }}$ is particularly pronounced for medium range porosities and this strongly depends on the upper and lower Wiener bounds.

It is therefore important to understand the dependence of the porosity, $f$, and the intrinsic refractive index, $n_{s}$, on these limits (Figure 4). The bounds are clearly very close to each other for very small porosities $\left(n_{\text {eff }}\right.$ approaches $\left.n_{s}\right)$ and for very high porosities $\left(n_{\text {eff }}\right.$ approaches $\left.n_{0}=1\right)$. The porosity maximising the separation of the Wiener bounds, $f_{\max }$, is of particular importance as it provides the highest resolution for the $S_{a}$ parameter. The fact that $f_{\max }$ is $<0.5$ for all $n_{s}$ renders the proposed method highly sensitive for the study of pharmaceutical tablets as these powder compacts have typically porosities $<0.5$.

The assumption of low terahertz absorption by the tablet samples for the derivation of Eq. 8 holds for the M01 and M02 samples. The definition of $S_{a}$, however, is broader and also allows for high absorption. The only adjustment required is the introduction of an effective refractive index that has a real (dispersion of terahertz waves) and imaginary (absorption of terahertz waves) part instead of the effective refractive index being confined to a real number (Peiponen and Gornov, 2006). The definition is, therefore, not limited to low absorption only. This fact is particularly important for the analysis of the M03 samples, where significant absorption of terahertz radiation by the formulation takes place. 
Table 4: Summary of the models used to determine the intrinsic refractive indices, $n_{s}$. More details about these models are provided in Markl et al. (2017b). The listed $n_{s}$ for M03 is the lumped intrinsic refractive index for the solid material. $L$ is the depolarisation factor used to describe the pore shape in the AB-EMA model. The references provide further details about the materials.

\begin{tabular}{ccccc}
\hline Set & & $n_{s}$ & Model & Ref \\
\hline M01 & FCC & 2.97 & $\begin{array}{c}\text { AB-EMA } \\
\text { M }\end{array}$ & Markl et al. (2017b) \\
& MCC & 1.86 & ZPA & Bawuah et al. (2016b) \\
M02 & API & 1.73 & & Ridgway et al. (2017) \\
& Complex & & AB-EMA & Markl et al. (2017a) \\
M03 & $\begin{array}{c}\text { formulation } \\
\text { form }\end{array}$ & & $L=0.35$ & \\
\hline
\end{tabular}
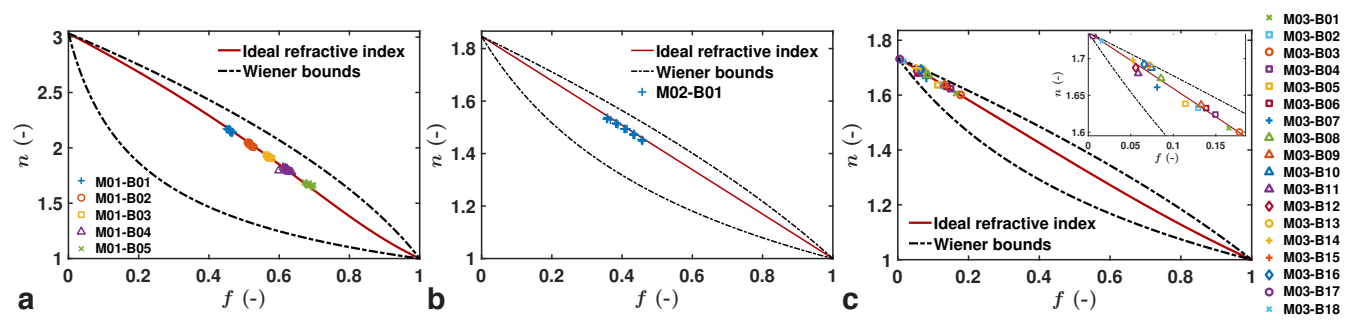

Figure 5: Refractive index, $n$, as a function of porosity, $f$, for (a) M01 (FCC tablets), (b) M02 (MCC and API tablets), and (c) M03 (complex formulation). The solid red line indicates the ideal refractive index for each material, which was calculated using the AB-EMA for (a,c) and ZPA for (b). (b) Shows

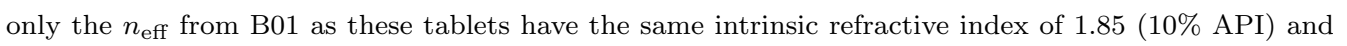
varied only in porosity.

\section{2. $S_{a}$ Parameter of the Different Materials}

The effective permittivity, $\varepsilon_{\text {eff }}$, and thus the effective refractive index, $n_{\text {eff }}$, always lie within the upper and lower limit of the Wiener bounds (Figure 5). The ideal refractive index was calculated from the model used to relate $n_{\text {eff }}$ and $f$ as well as to determine $n_{s}$. Here, we employ two different models for this purpose: the anisotropic Bruggeman EMA (AB-EMA) and the ZPA. The used models as well as the intrinsic refractive index values are summarised in Table 4 for the three different sets. Besides the use of these models to determine the intrinsic refractive indices and to predict the porosity from terahertz measurements, they were utilised to calculate the ideal refractive index required for the 
calculation of the $S_{a}$ parameter.

The AB-EMA was applied for M01 and M03 as it outperformed other EMA models as well as ZPA due to the fact that it accounts for a non-spherical shape of the inclusions and solid material by a depolarisation factor (Markl et al., 2017b). This depolarisation factor was estimated to be $L=0.21$ for M01, which can be related to a needle-like shape of the pores with an aspect ratio of 1.7 (ratio between the dimensions in $x$ - and $z$-direction). This means that the pores have a preferred orientation perpendicular to the compaction direction, and thus parallel to the $\mathbf{E}$ vector. The AB-EMA exhibits a non-linear dependence of the refractive index and the porosity (Figure $5 \mathrm{a}$ and c), which is not the case for the ZPA (Figure 5b). The depolarisation factor of M03 was $L=0.35$, which is very close to that of spherical shaped pores $(L=1 / 3)$ and the performance of the AB-EMA model was only slightly better than when using ZPA.

The M02 set consisted of several batches with varying porosity, thickness and API concentration. Since these samples are three-phase systems, the MCC and API particles were considered as one solid material. The lumped intrinsic refractive index, $n_{s, \text { lumped }}$, is not a constant for these samples as they vary in their composition (batches B03 and B04). The dependence of $n_{s, \text { lumped }}$ on the API concentration, $\phi$, is accounted for by

$$
n_{s, \text { lumped }}(\phi)=\left(n_{s, \mathrm{API}}-n_{\mathrm{s}, \mathrm{MCC}}\right) \phi+n_{s, \mathrm{MCC}} .
$$

$n_{s, \mathrm{API}}$ and $n_{\mathrm{s}, \mathrm{MCC}}$ are given in Table 4 and they were determined by the ZPA for a three-phase system following the approach presented in Bawuah et al. (2016b).

Figure 5b only depicts the data of M02-B01, which has a constant composition $(90 \%$ MCC and 10\% API) and only varies in terms of its porosity. The ideal refractive index changes with the composition leading to varying intersection points with the $y$-axis ranging from $1.73(100 \% \mathrm{API})$ to 1.86 (100\% MCC).

On the contrary, the M03 samples differed only in their microstructure and there were no changes in their composition. The lumped intrinsic refractive index $\left(n_{s, \text { lumped }}=1.74\right)$ was thus constant across all 18 batches.

The $S_{a}$ parameter of the M01 samples reveals that the structure changes with increasing porosity (Figure 6). A parallel arrangement of the FCC material and the pores becomes emphasised for a larger pore space, which is fully in line with the results in 


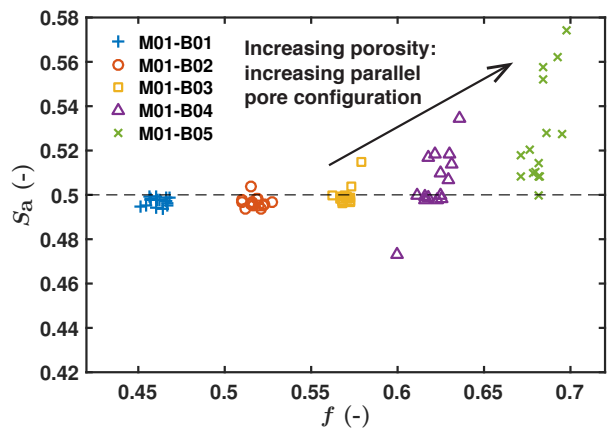

Figure 6: $S_{a}$ as a function of the porosity, $f$, for set M01 (FCC tablets). $n_{s}$ and the used model are provided in Table 4.

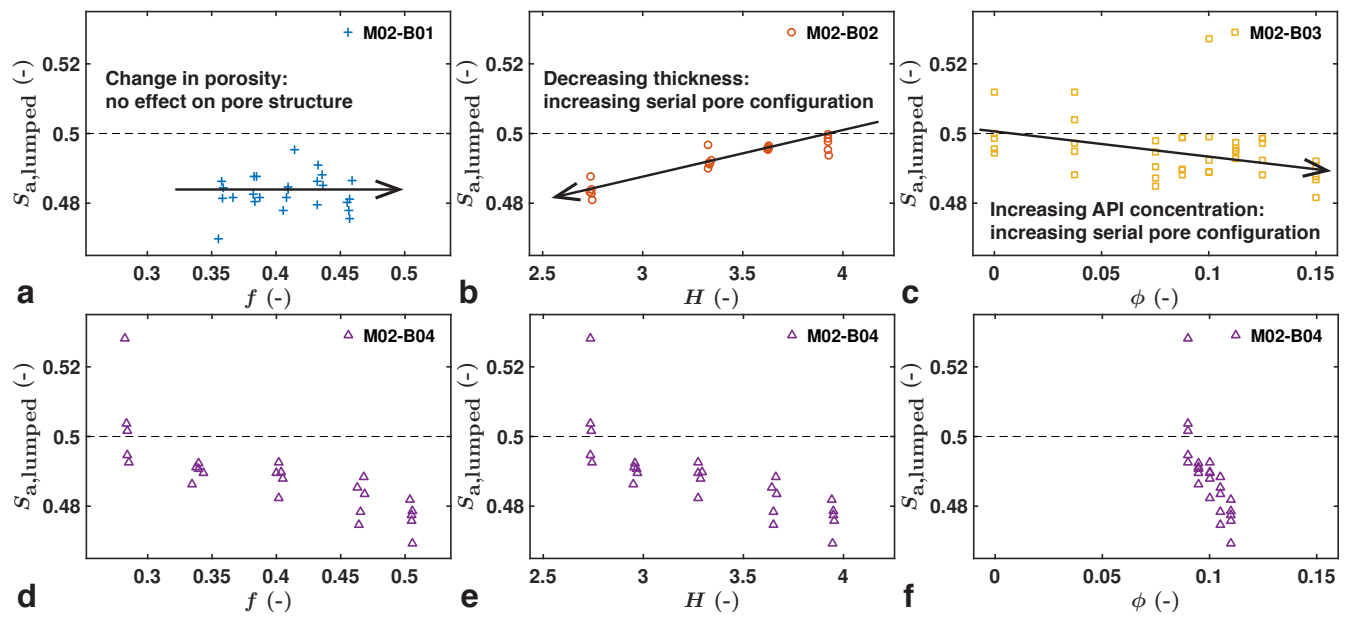

Figure 7: $S_{a}$ as a function of (a,d) the porosity, $f,(\mathrm{~b}, \mathrm{e})$ the tablet thickness, $H$, and $(\mathrm{c}, \mathrm{f})$ the API concentration, $\phi$, for set M02 (MCC/API tablets). Only one variable at a time was varied for results of the batches in the top row $(\mathrm{a}, \mathrm{b}, \mathrm{c})$. The bottom row $(\mathrm{d}, \mathrm{e}, \mathrm{f})$ shows the results from batch M02-B04, where all three variables were changed simultaneously. Eq. 20 was applied to calculate the intrinsic refractive index, $n_{s, \text { lumped }}(\phi)$, as a function of $\phi$.

Markl et al. (2017b). Although the FCC particles have a very large intraparticle porosity the anisotropic pore structure is primarily attributed to the interparticle voids that are formed during the compaction of the powder, as the FCC particles are known to be mechanically stable under compaction.

The results of a three-phase system (M02 samples) are depicted in Figure 7. Only 

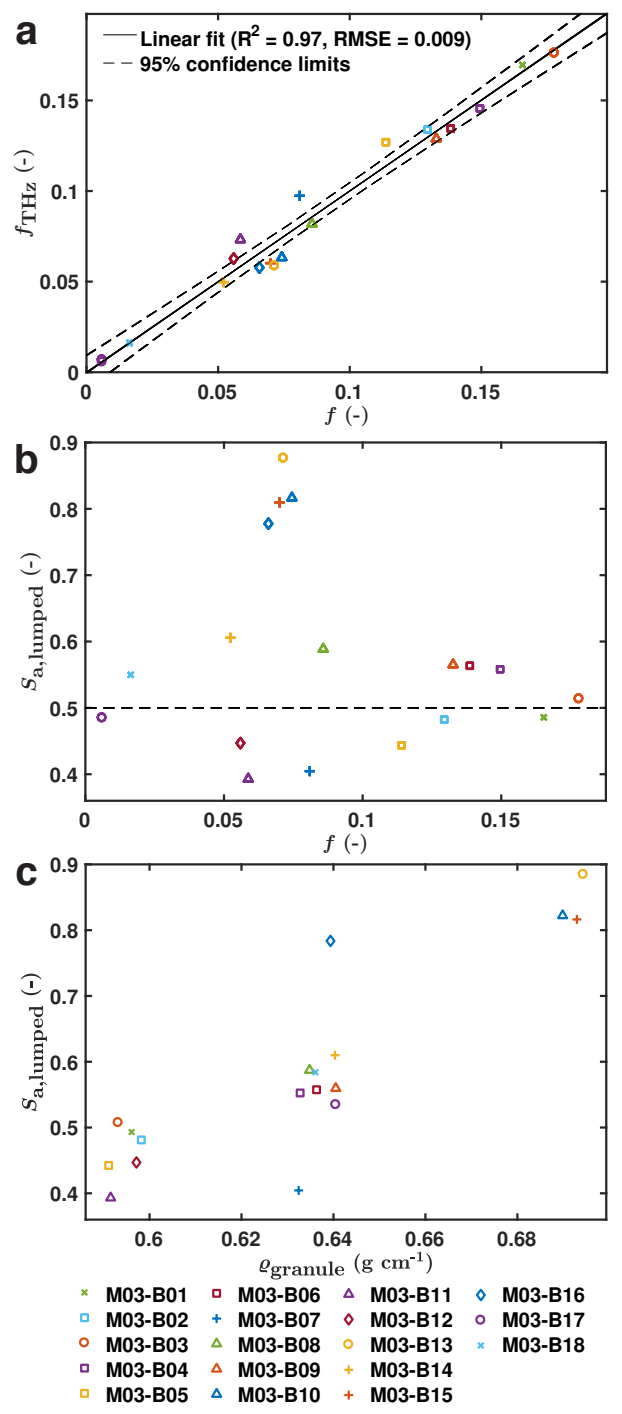

Figure 8: Results from terahertz measurements and analysis of the M03 set (complex formulation). (a) Porosity predicted from the terahertz measurements, $f_{\mathrm{THz}}$, as a function of the porosity determined by Eq. 19, (b) and (c) depict the $S_{a}$ parameter depending on the porosity and the granule density, respectively. AB-EMA was used to determine the intrinsic refractive index of the lumped solid material

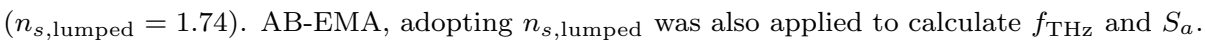
one parameter was varied at a time for the batches M02-B01 (porosity, Figure 7a), M02-B02 (tablet thickness, Figure 7b) and M02-B03 (API concentration, Figure 7c), 
whereas all three variables were modified for batch M02-B04 (Figure 7d-f). Surprisingly, the porosity does not considerably affect the arrangement of the solid material/pores construct (Figure 7a), although the overall configuration is a serial connection of the two phases. On the contrary, tablet thickness causes a change of the structure as a thinner tablet yields a more pronounced serial arrangement of solid material and pores (Figure $7 \mathrm{~b}$ ). The thicker the tablet the more randomly arranged the pores are. An increase of API particles in the powder compact has an opposite effect on the structure: the higher the API concentration, the more pronounced is a serial arrangement of solid material and air inclusions (Figure 7c). This is mostly attributed to the significantly different particle size of indomethacin $(\approx 13 \mu \mathrm{m})$ compared to that of $\mathrm{MCC}(\approx 50 \mu \mathrm{m})$. The increase in the API concentration thus causes a bimodal particle size distribution. It is well-known that in the majority of cases a smaller size of particles yields a higher tablet strength as the smaller particles provide a larger total surface area for bonding than the larger particles (Sun and Grant, 2001). However, a multimodal particle size distribution also affects the pore structure as smaller particles fit within the pores between larger particles, which eventually affects the arrangement of the solid phase and the pores.

Moreover, the most significant changes in the pore structure were observed when altering porosity, tablet thickness and API concentration simultaneously (Figure 7d-f). This yields a serial arrangement of solid material and pores for the highest values of each factor. This particularly highlights the interrelationship between these factors and the microstructure of the powder compact. The orientation and shape of the pores cannot be controlled by one single variable and it is the result of the initial particle properties as well as the process settings.

The M03 batches were produced for a production-scale DoE with its main objective to study the impact of three granulation states and one compaction factor on the disintegration as well as the dissolution performance of the tablets. Markl et al. (2017a) recently demonstrated how the terahertz measurements of the M03 batches can be used to determine the disintegration time and the API dissolved after a certain time period. This study also revealed that there is a good correlation between the terahertz effective refractive index, $n_{\text {eff }}$, and the solid fraction, i.e. porosity calculated from the true density values of each constituent and the bulk density (Eq. 19). The anisotropic Bruggeman 
model was used in this study to compute the porosity from the terahertz measurements, $f_{\mathrm{THz}}$ (Figure 8a). Even though the range of porosities between samples is very small $(<0.18)$ and the formulation is highly complex (four excipients and one API), the correlation between $f_{\mathrm{THz}}$ and $f$ is excellent $\left(R^{2}=0.97\right)$. The very small porosities of these samples render the $S_{a}$ parameter very sensitive to minor deviations of the $n_{\mathrm{eff}}$ from the ideal refractive index. This is also reflected by Figure $5 \mathrm{c}$, where the span between the upper and lower limits of the Wiener bounds is narrow compared to that around $f_{\max }=0.37$ (porosity maximising the Wiener bounds as denoted in Eq. 17). The $S_{a}$ parameter ranges from $0.39-0.88$ and there are four outliers around $S_{a}=0.8$ (Figure $8 \mathrm{~b})$. These outliers correspond to the batches with the most dense granules except for M03-B16 which is a batch with a slightly smaller granule density. We show here, for the first time, how the granule density impacts the pore structure that affects the disintegration process. The structure of the tablets thus clearly depend on the density of the granules (Figure 8c). The effect of the granule density on the compaction process is three-fold: i) initial granules rearrangement, ii) deformation potential, and iii) degree of fragmentation of the granules.

The data suggest that the degree of fragmentation of the granules increases with decreasing granule density and low dense granules are, therefore, acting as a more friable material. Fragmentation of the granules results in smaller particles, which leads to a larger total surface area for the formation of stronger bonds. In addition, the small particles fill the larger pore spaces between larger particles/granules, which has a similar effect on the pore structure as the increase of the API concentration for the M02 samples (Figure 7c). The very low granule density yields a serial arrangement of the solid phase and pores, whereas more dense granules cause a highly parallel arrangement of the two phases. The highly dense granules have a preference to deform rather than fragment and thus keep their integrity to some extent during compaction. Consequently, the tablet can be described as a large aggregate of the original granules.

The total pore space is therefore constructed by the structure between and within granules. The breakdown or collapse of the granules thus may also result in a less open pore structure in the tablet and it will impact the tablet permeability (Wikberg and Alderborn, 1990) and eventually the disintegration as well as dissolution performance. 
However, the disintegration time and also the API dissolved after a certain period of time is a function of the intragranular and intergranular pore structure as well as of the surface area of agglomerated particles that are formed when the compact breaks apart during disintegration (Markl et al., 2017a; van den Ban and Goodwin, 2017).

\section{Conclusions}

This study presents a new parameter to quantify anisotropy of porous media on the basis of terahertz transmission measurements. The developed concept is not only limited to the analysis of terahertz measurements, it can also be applied to data from other techniques, such as ultrasound and dielectric spectroscopy. The validity and importance of the proposed $S_{a}$ parameter was demonstrated for three different data sets ranging from a simple formulation to a complex tablet composition that is highly relevant for the industry. The results of the simple formulation (set M01) confirmed the observations from a previous study, which revealed that the pores are preferably orientated perpendicular to the compaction direction and that this phenomenon becomes more pronounced with increasing porosity.

The results of the second set of samples (set M02) revealed that a change in the particle size distribution of the initial powder impacted the structure of the finished tablet as the smaller particles filled the pores between larger particles. This could be observed by increasing the API concentration (smaller particles), which caused an increase of the serial arrangement of the solid phase and the pores.

The analysis of the pore structure of the complex formulation (set M03) indicated that a high granule density causes a significant change in the pore structure, i.e. the pores and solid material structure exhibits a parallel arrangement for granules with high density. This strong correlation between the pore structure and the granule density supports the conclusion from the formulation M02 that material properties considerably impact the pore architecture. This study clearly demonstrates that the particle size and granule density are of particular importance for the configuration of the pores in the finished product. However, we want to stress here that the pore structure cannot be controlled by one single variable. It is the result of a complex interrelationship between raw material attributes (e.g. particle size and shape) and process settings (e.g. compression pressure 
and speed) as well as process configuration (e.g. punch geometry and die size). The geometry and die size particularly influence the microstructure at the edges of the tablet, which would result in a change of the $S_{a}$ parameter at the edge compared to that in the tablet centre (as performed in this study). In future, transmitted terahertz waveforms could be acquired via a raster-scan to form an image of the entire tablet, which facilitates the investigation of structural variations within a tablet.

We have shown how terahertz transmission measurements of pharmaceutical powder compacts coupled with the $S_{a}$ parameter provide an approach to gain new insights into the pore structure. This will eventually lead to a better understanding of the relationship between the structure and the disintegration as well as the dissolution performance of pharmaceutical tablets.

\section{References}

van den Ban, S., Goodwin, D.J., 2017. The Impact of Granule Density on Tabletting and Pharmaceutical Product Performance. Pharm. Res. 34, 1002-1011.

Bawuah, P., Chakraborty, M., Ervasti, T., Zeitler, J.A., Ketolainen, J., Gane, P.A.C., Peiponen, K.E., 2016a. A structure parameter for porous pharmaceutical tablets obtained with the aid of Wiener bounds for effective permittivity and terahertz time-delay measurement. Int. J. Pharm. 506, 87-92.

Bawuah, P., Tan, N., Tweneboah, S.N.A., Ervasti, T., Zeitler, J.A., Ketolainen, J., Peiponen, K.E., 2016b. Terahertz study on porosity and mass fraction of active pharmaceutical ingredient of pharmaceutical tablets. Eur. J. Pharm. Biopharm. 105, 122-133.

Berg, C.F., 2014. Permeability Description by Characteristic Length, Tortuosity, Constriction and Porosity. Transp. Porous Media 103, 381-400.

Eiliazadeh, B., Briscoe, B.J., Sheng, Y., Pitt, K., 2003. Investigating Density Distributions for Tablets of Different Geometry During the Compaction of Pharmaceuticals. Part. Sci. Technol. 21, 303-316.

Eiliazadeh, B., Pitt, K., Briscoe, B., 2004. Effects of punch geometry on powder movement during pharmaceutical tabletting processes. Int. J. Solids Struct. 41, 5967-5977.

Jepsen, P.U., Møller, U., Merbold, H., 2007. Investigation of aqueous alcohol and sugar solutions with reflection terahertz time-domain spectroscopy. Opt. Express 15, 14717.

Kadlec, C., Kadlec, F., Kuzel, P., Blary, K., Mounaix, P., 2008. Materials with on-demand refractive indices in the terahertz range. Opt. Lett. 33, 2275.

Krischer, O., Kast, W., 1978. Trocknungstechnik. Die wissenschaftlichen Grundlagen der Trocknungstecknik, Springer Berlin Heidelberg, Berlin, Heidelberg.

Li, R., Zeitler, J.A., Tomerini, D., Parrott, E.P.J., Gladden, L.F., Day, G.M., 2010. A study into the effect of subtle structural details and disorder on the terahertz spectrum of crystalline benzoic acid. Phys. Chem. Chem. Phys. 12, 5329. 
Liu, G., Zhang, M., Ridgway, C., Gane, P., 2014. Spontaneous Inertial Imbibition in Porous Media Using a Fractal Representation of Pore Wall Rugosity. Transp. Porous Media 104, 231-251.

Markl, D., Sauerwein, J., Goodwin, D.J., van den Ban, S., Zeitler, J.A., 2017a. Non-destructive Determination of Disintegration Time and Dissolution in Immediate Release Tablets by Terahertz Transmission Measurements. Pharm. Res. 34, 1012-1022.

Markl, D., Wang, P., Ridgway, C., Karttunen, A.P., Chakraborty, M., Bawuah, P., Pääkkönen, P., Gane, P., Ketolainen, J., Peiponen, K.E., Zeitler, J.A., 2017b. Characterization of the Pore Structure of Functionalized Calcium Carbonate Tablets by Terahertz Time-Domain Spectroscopy and X-Ray Computed Microtomography. J. Pharm. Sci. 106, 1586-1595.

Markl, D., Zeitler, J.A., 2017. A Review of Disintegration Mechanisms and Measurement Techniques. Pharm. Res. 34, 890-917.

Moe, D.V., Rippie, E.G., 1997. Nondestructive viscoelastic analysis of anisotropy in compressed tablets. J. Pharm. Sci. 86, 26-32.

Mullarney, M.P., Hancock, B.C., 2006. Mechanical property anisotropy of pharmaceutical excipient compacts. Int. J. Pharm. 314, 9-14.

Nyström, C., Alderborn, G., Duberg, M., Karehill, P.G., 2008. Bonding Surface area and Bonding Mechanism-Two Important Factors fir the Understanding of Powder Comparability. Drug Dev. Ind. Pharm. 19, 2143-2196.

Parrott, E.P.J., Zeitler, J.A., Friščić, T., Pepper, M., Jones, W., Day, G.M., Gladden, L.F., 2009. Testing the Sensitivity of Terahertz Spectroscopy to Changes in Molecular and Supramolecular Structure: A Study of Structurally Similar Cocrystals. Cryst. Growth \& Des. 9, 1452-1460.

Peiponen, K.E., Gornov, E., 2006. Description of Wiener bounds of multicomponent composites by barycentric coordinates. Opt. Lett. 31, 2202-2204.

Ridgway, C., Bawuah, P., Markl, D., Zeitler, J.A., Ketolainen, J., Peiponen, K.E., Gane, P., 2017. On the role of API in determining porosity, pore structure and bulk modulus of the skeletal material in pharmaceutical tablets formed with MCC as sole excipient. Int. J. Pharm. 526, 321-331.

Schoelkopf, 2000. Influence of inertia on liquid absorption into paper coating structures. Nord. Pulp Pap. Res. J. 15, 422-430.

Sun, C., Grant, D.J.W., 2001. Effects of initial particle size on the tableting properties of l-lysine monohydrochloride dihydrate powder. Int. J. Pharm. 215, 221-228.

Tuononen, H., Fukunaga, K., Kuosmanen, M., Ketolainen, J., Peiponen, K.E., 2010. Wiener Bounds for Complex Permittivity in Terahertz Spectroscopy: Case Study of Two-Phase Pharmaceutical Tablets. Appl. Spectrosc. 64, 127-131.

Wiener, O., 1912. Die Mittelwertsätze für Kraft, Polarisation und Energie. Abhandlungen der Math. Klasse der Königlich-Sächsischen Gesellschaft der Wissenschaften 32, 509-604.

Wikberg, M., Alderborn, G., 1990. Compression characteristics of granulated materials II. Evaluation of granule fragmentation during compression by tablet permeability and porosity measurements. Int. J. Pharm. 62, 229-241.

Yassin, S., Goodwin, D.J., Anderson, A., Sibik, J., Wilson, D.I., Gladden, L.F., Zeitler, J.A., 2015a. The 
459 Yassin, S., Su, K., Lin, H., Gladden, L.F., Zeitler, J.A., 2015b. Diffusion and Swelling Measurements in 